\title{
Effect of estrous expression on timing and failure of ovulation of Holstein dairy cows using automated activity monitors
}

\author{
Tracy A. Burnett, Liam Polsky, Manveen Kaur, and Ronaldo L. A. Cerri ${ }^{1}$ \\ Faculty of Land and Food Systems, University of British Columbia, Vancouver, V6T 1Z4, Canada
}

\begin{abstract}
The aim of this study was to determine if estrous expression, as measured by an automated activity monitor (AAM), affects timing and failure of ovulation of lactating Holstein dairy cows. Cows were equipped with 2 AAM, 1 neck-mounted $\left(\mathrm{AAM}_{\mathrm{C}}\right)$ and 1 leg-mounted $\left(\mathrm{AAM}_{\mathrm{L}}\right)$, by $10 \mathrm{~d}$ postpartum and enrolled into the trial when their activity crossed the alert threshold on the $\mathrm{AAM}_{\mathrm{C}}$. A total of 850 episodes of estrus from 293 different cows were used for this study. When cows were enrolled, their ovaries were scanned by transrectal ultrasonography and gait and body condition scored. Ovaries of cows detected in estrus were scanned twice daily for a maximum of $3 \mathrm{~d}$ to determine the disappearance of the preovulatory follicle (ovulation) and the interval from estrus to ovulation was calculated. Physical activity data recorded from the AAM were used to determine estrus behavior using 2 traits: (1) peak activity and (2) duration. Peak activity was only available for the $\mathrm{AAM}_{\mathrm{L}}$. Peak activity was defined as the maximum activity during an estrus episode. Duration of estrus was defined as the time the activity of the cow exceeded threshold values set by the AAM software. The $\mathrm{AAM}_{\mathrm{C}}$ correctly identified $87.8 \%$ of the estrus alerts, with $12.2 \%$ false positives. The average ( \pm standard deviation) intervals from activity alert to ovulation were $25.8 \pm 10.2$ and $24.7 \pm 9.3 \mathrm{~h}$ for the $\mathrm{AAM}_{\mathrm{C}}$ and $\mathrm{AAM}_{\mathrm{L}}$, respectively. Changes in estrous expression were associated with differences in the interval from alert to ovulation. Cows with short intervals to ovulation were found to have less intense estrous expression than cows with medium and long length intervals to ovulation using the $\mathrm{AAM}_{\mathrm{C}}$, whereas using the $\mathrm{AAM}_{\mathrm{L}}$, cows with short intervals to ovulation exhibited less intense estrous expression than cows with medium but the same as those with long intervals to ovulation. Furthermore, irrespective of the AAM, estrus events with less estrous expression had increased odds of hav-
\end{abstract}

Received June 1, 2018.

Accepted July 30, 2018.

${ }^{1}$ Corresponding author: ronaldo.cerri@ubc.ca ing a short interval to ovulation (below the median of $20 \mathrm{~h}$ ) when compared with those having greater estrous expression (2.6 and 1.9 increased odds for the $\mathrm{AAM}_{\mathrm{C}}$ and $\mathrm{AAM}_{\mathrm{L}}$, respectively). Ovulation failure was affected by estrous expression because estrus events with greater peak activity or longer duration had reduced ovulation failure compared with those with less estrous expression $\left(\mathrm{AAM}_{\mathrm{C}}\right.$ peak activity: $1.9 \pm 1.4$ vs. $9.5 \pm$ $1.7 \% ; \mathrm{AAM}_{\mathrm{L}}$ peak activity: $2.3 \pm 1.4$ vs. $6.2 \pm 1.5 \%$; $\mathrm{AAM}_{\mathrm{C}}$ duration: $2.1 \pm 1.4$ vs. $8.9 \pm 1.7 \%$ ). In addition, cows with more estrous expression had greater pregnancy per artificial insemination than those with less estrous expression with both the $\mathrm{AAM}_{\mathrm{C}}(42.3 \pm$ 0.4 vs. $31.7 \pm 0.4 \%)$ and the $\mathrm{AAM}_{\mathrm{L}}(43.1 \pm 0.4$ vs. 36.3 $\pm 0.4 \%)$. Pregnancy per artificial insemination results were consistent even when removing cows that failed to ovulate. In conclusion, expression of estrus was highly associated with ovulation timing, ovulation failure, and fertility when using 2 different AAM. Cows with greater estrous expression have longer intervals from activity alert to ovulation, experience less ovulation failure, and have greater pregnancy per artificial insemination.

Key words: ovulation, automated activity monitor, estrus

\section{INTRODUCTION}

Timing of AI relative to ovulation is important for successful fertilization. Inseminations occurring too long after the onset of estrus will not permit sufficient time for capacitation of the spermatozoa, resulting in the fertilization of poor quality oocytes, whereas insemination too early will result in the death of many spermatozoa before the oocyte arrives at the ampulla for fertilization (Saacke et al., 2000; Dalton et al., 2001).

Previous reports have discussed the optimal timing of AI relative to estrus behaviors. The most optimal interval for AI after the onset of visually detected estrus was 7 to $12 \mathrm{~h}$ (Hall et al., 1959). Another study reported the greatest conception rates for inseminations occurring 4 to $12 \mathrm{~h}$ after the onset of estrus using a pressuresensing system (Dransfield et al., 1998). Using an automated activity monitor (AAM), Stevenson et al. 
(2014) reported a difference between primiparous and multiparous cows, where the optimal time for AI relative to onset of estrus was 13 to $16 \mathrm{~h}$ postalert, whereas primiparous cows had relatively stable conception risks when being inseminated any time between 0 to $16 \mathrm{~h}$ postalert. Using visual detection, Van Eerdenburg et al. (2002) reported that cows that ovulated between 0 and $24 \mathrm{~h}$ post-AI had greater conception rates and also had more estrous expression than those that ovulated before insemination or later than $24 \mathrm{~h}$.

High-producing cows have reduced estrous expression (Lopez et al., 2004; Rivera et al., 2010), which can be further compromised by current management practices in which cows are kept in freestall barns with concrete flooring and little or no access to dirt lots (Stevenson, 2001). Decreased estrous expression has not only made the detection of cows for AI more difficult, but has also been seen to affect the fertility of the cow. Dransfield et al. (1998) reported that cows that stood to be mounted more times per estrus had greater conception rates. Performing standing estrus (Bijker et al., 2015), having increased intensity of estrus (Madureira et al., 2015), and having increased walking activity (López-Gatius et al., 2005) have all individually been shown to have better pregnancy success in dairy cows.

Other common factors affecting fertility are issues with physical health such as lameness and BCS. Reduced BCS is associated with reduced conception rates at first AI postpartum, more days open, and postponed resumption of cyclicity resulting in a longer interval from parturition to first estrus and first service (Roche et al., 2009). Carryover effects of reduced BCS during the transition period have been demonstrated to be detrimental even after the voluntary waiting period, where the likelihood of pregnancy has been found to be reduced with each 0.5-unit decrease of BCS (Buckley et al., 2003; Roche et al., 2007). Lameness also is associated with less pregnancy per AI (Burnett et al., 2017), and increased number of inseminations per conception (Hernandez et al., 2001; Melendez et al., 2003), days to conception (Burnett et al., 2017), and prevalence of ovarian cysts (Melendez et al., 2003). It is not well understood how expression of estrus, BCS, and lameness affect fertility. One potential cause may be from disruptions of the events that lead to ovulation. In the present study, we aimed to determine if the reductions in fertility that are found with lesser estrous expression, as well as with lameness and BCS, are associated with the interval from activity alert to ovulation using AAM.

The main goal of this project was to determine the effect of estrous expression, as measured by 2 different AAM, on ovulation timing and ovulation failure. We hypothesized that decreased estrous expression and compromised physical health (i.e., poor BCS and lameness) would increase the proportion of cows with ovulation failure and those ovulating outside the ideal timing relative to the onset of estrus and consequently insemination.

\section{MATERIALS AND METHODS}

This experiment was conducted at the University of British Columbia's Dairy Education and Research Centre, Agassiz, BC, Canada. All procedures were approved by the Animal Care Committee of the University of British Columbia. Animals used in this experiment were cared for as outlined by the guidelines provided by the Canadian Council of Animal Care (2009).

\section{Animals and Housing}

A total of 850 episodes of estrus were used from 293 high-producing Holstein dairy cows in this study; an average of (mean $\pm \mathrm{SD}$ ) $2.5 \pm 1.7$ episodes were used per cow. Cows had a mean DIM of $103 \pm 56$, lactation of $2.7 \pm 1.7$, BCS of $2.80 \pm 0.20$, gait score (GS) of $2.2 \pm 0.8$, hock score of $1.3 \pm 0.5$, and a 305 -d matureequivalent yield of $12,500 \pm 1,900 \mathrm{~kg}$ of milk. Cows were housed in a naturally ventilated wooden-framed barn with a freestall design, equipped with deep sandbedded stalls. Cows were milked twice daily at 0500 and $1500 \mathrm{~h}$ through a conventional milking parlor. Fresh TMR was delivered twice daily at approximately 0700 and $1600 \mathrm{~h}$. The TMR was formulated following the NRC guidelines (NRC, 2001) to meet or exceed the requirements of a $620-\mathrm{kg}$ Holstein cow producing 40 $\mathrm{kg} / \mathrm{d}$ of $3.5 \%$ FCM. All cows had ad libitum access to both feed and water.

\section{Experimental Design}

This experiment was an observational cohort study. Cows were enrolled onto the study when they crossed the alert threshold, set by the company recommendations for the collar-mounted AAM $\left(\mathbf{A} \mathbf{A} \mathbf{M}_{\mathbf{C}}\right)$. At enrolment, cows were scored for body condition, gait, and hock, and scanned by transrectal ultrasonography to determine ovarian structures using an ultrasound (Ibex Pro, E.I. Medical Imaging, Loveland, CO) with a 7.5$\mathrm{MHz}$ linear-array probe. Inclusion criteria for the present study was that cows had to be greater than or equal to 30 DIM and have an estrus alert on the $\mathrm{AAM}_{\mathrm{C}}$. The AAM were checked twice daily during milking times for cows in estrus. Cows classified as in estrus were then monitored for ovulation using ultrasonography after each milking $(2 \times$ per d) at approximately 0800 and $1700 \mathrm{~h}$ for a maximum of 6 times (approximately $60 \mathrm{~h}$ after the first ultrasound). 
Each cow was equipped with an $\mathrm{AAM}_{\mathrm{C}}$ (Heatime, H-Tags, SCR Engineers, Netanya, Israel) before $10 \mathrm{~d}$ postcalving and a leg-mounted AAM $\left(\mathbf{A A M}_{\mathbf{L}}\right.$ : Boumatic Heat-seeker-TX, Boumatic Dairy Equipment Co., Madison, WI) on the day of calving of their first lactation.

\section{Determination of Estrus, Ovulation, and Ovulation Times}

Cows were classified to be in estrus if they had at least one dominant pre-ovulatory follicle greater than $15 \mathrm{~mm}$ and an absence of a large corpus luteum $(>20$ $\mathrm{mm}$ ) at the time of the estrus alert. Ovulation was determined by the disappearance of the dominant preovulatory follicle; the time of ovulation was determined as the median time between the ultrasound where the pre-ovulatory follicle disappeared and the ultrasound preceding its disappearance. For estrus events where ovulation occurred after the maximum of 6 ultrasounds, ovulation time was calculated as the time of the last ultrasound plus $7.75 \mathrm{~h}$ (the average median hours between all ultrasounds). Intervals from activity alert to ovulation were calculated as the time from when an alert on the respective AAM occurred until the time of ovulation. Intervals to ovulation were classified into 12 -h periods as short $(<20 \mathrm{~h})$, medium $(20-31 \mathrm{~h})$, or long ( $\geq 32 \mathrm{~h}) ; 12$-h blocks were used because assessment for ovulation was only carried out twice daily.

All cows with estrus alerts, including false alerts, were scanned using an ultrasound $7 \mathrm{~d}$ after the alert for the presence of a new corpus luteum, which was used for confirmation of estrus and ovulation classifications. For true estrus alerts where ovulation was detected, a new corpus luteum was used to confirm ovulation. For true estrus alerts where ovulation was not detected by the maximum of 6 ovarian ultrasound checks, the presence of a new corpus luteum was used to determine late ovulations and the absence of a new corpus luteum was indicative of failed ovulation. For false estrus alerts, the absence of a new corpus luteum was used to confirm that the estrus alert was correctly classified as false.

\section{Expression of Estrus}

Physical activity was continuously monitored using both AAM. The threshold was set at an activity index of 35 for the $\mathrm{AAM}_{\mathrm{C}}$ and at a relative increase in activity of $180 \%$ for the $\mathrm{AAM}_{\mathrm{L}}$; an index activity of 35 equates approximately to a 6 -standard deviation increase in activity in relation to baseline within the $\mathrm{AAM}_{\mathrm{C}}$ system. Two traits, previously used by Madureira et al. (2015), were used to describe the expression of estrus: (1) peak activity and (2) duration. Peak activity was defined as the maximum activity (in index or percentage relative increase for $\mathrm{AAM}_{\mathrm{C}}$ and $\mathrm{AAM}_{\mathrm{L}}$, respectively) during an episode of estrus. Duration of estrus was defined as the time the activity of the cow exceeded threshold values set by the AAM software. The term estrous expression will be used to encompass both traits unless specified. For the $\mathrm{AAM}_{\mathrm{L}}$, only peak activity could be calculated, although both peak activity and duration were calculated for the $\mathrm{AAM}_{\mathrm{C}}$. Peak activity and duration of estrus were categorized into groups above and below their respective medians (peak activity $\mathrm{AAM}_{\mathrm{C}}$ : high $\geq 80$ index, low $<80$ index; peak activity $\mathrm{AAM}_{\mathrm{L}}$ : high $\geq 331$ relative increase $(\%)$, low $<331$ relative increase (\%); duration $\mathrm{AAM}_{\mathrm{C}}$ : long $\geq 12 \mathrm{~h}$, short $<12 \mathrm{~h}$ ).

\section{Health Scoring}

All cows were scored for body condition, gait, and hock at the time of enrollment in the study. Body condition was scored on a 5-point scale from thin (1) to obese (5) as outlined by Edmonson et al. (1989). Cows were later categorized as thin $(\leq 2.50)$, average ( $=2.75)$, or moderate $(\geq 3.00)$. Lameness was scored on a 5-point scale from sound (1) to severely lame (5) as outlined by Flower and Weary (2006). Cows were later classified as sound $(\leq 2)$ and lame $(\geq 3)$. Hock lesions were scored on a 4-point scale modified from the Hock Assessment Chart for Cattle developed by Cornell Cooperative Extension (http://hdl.handle.net/1813/ 36913) as follows: (1) no swelling and without balding, (2) minor swelling without balding, (3) minor to moderate swelling with bald area, and (4) severe swelling with bald area, with or without broken or scabbed skin. As the incidence of hock lesions of $\geq 3$ was only $1.8 \%$, not enough variation was present, and the variable was removed from the study.

Milk weights were recorded automatically twice daily at milking through a conventional milking parlor (Boumatic Dairy Equipment Co.) and the data were automatically transferred and stored on Dairy Comp 305 software (Valley Agricultural Software, Tulare, CA). Health and production information was collected by the dairy herd personnel with the assistance of the herd veterinarian, and confirmed and recorded by the project leader using the on-farm Dairy Comp 305 software.

\section{Ultrasonography and Pregnancy Diagnosis}

Ovaries of cows in estrus were examined by ultrasound (Ibex Pro, E.I. Medical Imaging) using a 7.5$\mathrm{MHz}$ linear-array rectal probe at enrolment, and twice daily, until a maximum of 6 palpations. Presence and diameter of the 3 largest follicles and corpora lutea 
were measured and recorded. Cows were inseminated at estrus using the $\mathrm{AM} / \mathrm{PM}$ rule in relation to when the estrus alert was triggered, the mean $( \pm \mathrm{SD})$ time from alert to breeding was $11.0 \pm 5.3$ and $10.1 \pm 6.5 \mathrm{~h}$ for the $\mathrm{AAM}_{\mathrm{C}}$ and $\mathrm{AAM}_{\mathrm{L}}$, respectively. The voluntary waiting period was 60 DIM and the cows had a mean number of inseminations of $2.6 \pm 1.7$, with a range from first to ninth service. All cows were examined by way of ultrasound for pregnancy at $36 \pm 7 \mathrm{~d}$ post-AI for the detection of an embryonic vesicle with a viable embryo (presence of heartbeat). Pregnancy diagnosis was carried out by the herd veterinarians.

\section{Statistical Analyses}

All statistical analyses in this study were carried out with SAS Studio (version 3.4, SAS Institute Inc., Cary, NC) with episode of estrus as the experimental unit. Prior to all analyses, data were checked for normality using the UNIVARIATE procedure and probability distribution plots. Due to collinearity, $\mathrm{AAM}_{\mathrm{C}}$ peak activity, $\mathrm{AAM}_{\mathrm{C}}$ duration, and $\mathrm{AAM}_{\mathrm{L}}$ peak activity were not used together in the same model as independent variables.

Peak activity and duration of estrus were used as continuous dependent variables and tested for the fixed effects of parity, BCS, GS, stage of lactation $(\leq 60$, $61-119$, and $\geq 120 \mathrm{DIM})$, interval to ovulation $(<20$, 20-31, and $\geq 31 \mathrm{~h}$ ), and false alerts using ANOVA with cow as a random effect using the MIXED procedure. Pregnancy per AI and ovulation failure were binomial dependent variables assessed using the GLIMMIX procedure with cow as the random effect, estrus event as the experimental unit and parity, BCS, GS, stage of lactation ( $\leq 60,61-119$, and $\geq 120$ DIM), and estrous expression $\left(\mathrm{AAM}_{\mathrm{C}}\right.$ peak activity, $\mathrm{AAM}_{\mathrm{C}}$ duration, or $\mathrm{AAM}_{\mathrm{L}}$ peak activity) as fixed effects. Peak activity and duration were categorized by the median for both $\mathrm{AAM}_{\mathrm{C}}$ (peak activity: high and low; duration: long and short) and $\mathrm{AAM}_{\mathrm{L}}$ (peak activity: high and low). The LOGISTIC procedure, with backward elimination using Wald's statistic criterion when $P<0.15$, was used to determine which independent variables (same as above) affected the occurrence of long and short intervals from activity alert to ovulation (using the median of $20 \mathrm{~h}$ ) and false alerts. Results from the MIXED and GLIMMIX procedures are presented as LSMEANS, whereas those from LOGISTIC are presented as odds ratios. Values were considered a tendency when having a $P$-value of $\leq 0.10$ and as statistically significant when $\leq 0.05$. Time of day of estrus alert was used as a covariate as previous research has reported it to affect the expression of estrus (Stevenson et al., 2014).

\section{RESULTS}

\section{Intervals from Activity Alert to Ovulation}

The average time from $\mathrm{AAM}_{\mathrm{C}}$ alert to ovulation was (mean $\pm \mathrm{SD}$ ) $25.8 \pm 10.2 \mathrm{~h}$, and the average time from $\mathrm{AAM}_{\mathrm{L}}$ alert to ovulation was $24.7 \pm 9.3 \mathrm{~h}$. Descriptive statistics of $\mathrm{AAM}_{\mathrm{C}}$ intervals to ovulation, $\mathrm{AAM}_{\mathrm{L}}$ intervals to ovulation, and AI to ovulation intervals are summarized in Table 1 . The average peak activity $\left(\mathrm{AAM}_{\mathrm{C}}\right.$ and $\left.\mathrm{AAM}_{\mathrm{L}}\right)$ and duration $\left(\mathrm{AAM}_{\mathrm{C}}\right)$ found in this study were $72.4 \pm 20.7$ index, $380 \pm 146$ relative increase (\%), and $11.1 \pm 5.4 \mathrm{~h}$, respectively.

Changes in estrous expression were associated with differences in intervals to ovulation. Cows with a short interval to ovulation exhibited less estrous expression than cows with medium and long length intervals to ovulation using the $\operatorname{AAM}_{\mathrm{C}}(P<0.001)$, whereas when using the $\mathrm{AAM}_{\mathrm{L}}$, cows with short intervals to ovulation performed less estrous expression than cows with medium but the same as those with long intervals to ovulation $(P=0.02)$. Data on estrous expression and intervals from activity alert to ovulation have been summarized in Table 2. When using the $\mathrm{AAM}_{\mathrm{C}}$, the odds of events of estrus with less peak activity or shorter duration having a short interval to ovulation (below the median of $20 \mathrm{~h}$ ) were 2.6 and 4.7 times greater than the odds of an estrus event with greater activity $(P<0.001)$ or longer duration $(P<0.001)$, respectively. When using the $\mathrm{AAM}_{\mathrm{L}}$, similar results were found, where the

Table 1. Descriptive statistics of ovulation timing variables

\begin{tabular}{lccccc}
\hline Factor $^{1}$ & $\mathrm{n}$ & $\begin{array}{c}\text { Mean interval } \\
\text { time }(\mathrm{h})\end{array}$ & $\mathrm{SD}$ & Minimum & Maximum \\
\hline AI-ovulation $^{\text {AAM }}$ & 426 & 15.0 & 8.8 & -8.0 & 62.7 \\
AAM alert-ovulation & 627 & 25.8 & 10.2 & -2.5 & 87.1 \\
AAM alert-AI & 498 & 11.0 & 5.2 & -19.3 & 23.5 \\
AAM $_{\mathrm{L}}$ & 499 & 24.7 & 9.2 & -0.2 & 89.8 \\
AAM alert-ovulation & 384 & 10.1 & 6.5 & -21.7 & 27.8 \\
AAM alert-AI & &
\end{tabular}

${ }^{1} \mathrm{AAM}_{\mathrm{C}}=$ collar-mounted automated activity monitor; $\mathrm{AAM}_{\mathrm{L}}=$ leg-mounted automated activity monitor. 
Table 2. Associations of intervals from activity alert to ovulation and estrous expression traits measured using 2 automated activity monitors (AAM)

\begin{tabular}{|c|c|c|c|c|c|c|c|}
\hline \multirow{2}{*}{ Factor } & \multicolumn{6}{|c|}{ Interval to ovulation $(\mathrm{h})$} & \multirow{2}{*}{$P$-value } \\
\hline & \multicolumn{2}{|c|}{$<20$} & \multicolumn{2}{|c|}{$20-31$} & \multicolumn{2}{|c|}{$\geq 32$} & \\
\hline Peak activity $\mathrm{AAM}_{\mathrm{C}}^{1}$ (index) & $69.9^{\mathrm{a}}$ & 1.6 & $80.7^{\mathrm{b}}$ & 1.0 & $78.4^{\mathrm{b}}$ & 1.9 & $<0.001$ \\
\hline Peak activity $\mathrm{AAM}_{\mathrm{L}}{ }^{1}$ (\%-relative increase) & $361.9^{\mathrm{a}}$ & 13.4 & $405.5^{\mathrm{b}}$ & 8.9 & $388.8^{\mathrm{ab}}$ & 18.1 & 0.02 \\
\hline Duration of estrus $\mathrm{AAM}_{\mathrm{C}}{ }^{2}(\mathrm{~h})$ & $10.1^{\mathrm{a}}$ & 0.4 & $12.9^{\mathrm{b}}$ & 0.3 & $13.3^{\mathrm{b}}$ & 0.5 & $<0.001$ \\
\hline
\end{tabular}

${ }^{\mathrm{a}, \mathrm{b}}$ Values with different superscripts in the same row are significantly different $(P<0.05)$.

${ }^{1}$ Peak activity was defined as the maximum activity during an episode of estrus. $\mathrm{AAM}_{\mathrm{C}}=$ collar-mounted automated activity monitor; $\mathrm{AAM}_{\mathrm{L}}$ $=$ leg-mounted automated activity monitor.

${ }^{2}$ Duration of estrus was defined as the time the activity of the cow exceeded threshold values set by the AAM software.

odds of an estrus event with lesser activity having a short interval to ovulation was 1.9 times greater than the odds of estrus events with increased peak activity $(P<0.01)$. The frequency of estrus events with low and high estrous expression in each of the ovulation interval categories has been summarized in Figure 1.

When using the $\mathrm{AAM}_{\mathrm{C}}$, estrus events occurring in early lactation (30-60 DIM) had 1.7 and 2.3 greater odds of having an interval to ovulation less than the median $(<20 \mathrm{~h})$ than those in mid $(61-119)$ or late lactation $(\geq 120 \mathrm{DIM})$, respectively $(P=0.01)$. When using the $\mathrm{AAM}_{\mathrm{L}}$, the odds of estrus events early in lactation having an interval to ovulation of $<20 \mathrm{~h}$ was 2.3 times greater than the odds of estrus events in late lactation $(P<0.01)$. In addition, the odds of a lame cow having an interval to ovulation $\geq 20 \mathrm{~h}$ were 1.9 times greater than the odds of a sound cow $(P=0.01)$. No effects of GS were found on intervals to ovulation when using the $\mathrm{AAM}_{\mathrm{L}}(P=0.12)$. No effects of BCS, parity, or the size of the pre-ovulatory follicle were found on intervals to ovulation for either monitor.

\section{Ovulation Failure and False Alerts}

Considering all the estrus alerts that appeared on the $\mathrm{AAM}_{\mathrm{C}}, 81.7 \%$ of these alerts resulted in ovulation. When excluding false alerts, $93.3 \%$ of the estrus alerts resulted in ovulation, equating to $6.7 \%$ ovulation failure. Ovulation failure was affected by estrous expression and parity but not by stage of lactation, BCS, GS, milk production, or the size of the pre-ovulatory follicle. Events of estrus with less estrous expression had increased ovulation failure, where $1.9 \pm 1.4 \%$ and $9.5 \pm 1.7 \%$ and $8.9 \pm 1.7 \%$ and $2.1 \pm 1.4 \%$ ovulation failure was found for high and low peak activity and short and long duration episodes of estrus using the $\mathrm{AMM}_{\mathrm{C}}$, respectively $(P<0.001)$. Using the $\mathrm{AAM}_{\mathrm{L}}$, the same relationship was found where events of estrus with less estrous expression experienced more ovulation failure ( $P=0.03$; Table 3$)$. Additionally, parity was found to affect ovulation failure, where multiparous cows had increased ovulation failure when compared with primiparous cows $(P=0.05)$. Ovulation failure results are summarized in Table 3.

The positive predictive value (the number of estrus alerts that correctly identified a cow in estrus divided by the total alerts on the monitor) for the $\mathrm{AAM}_{\mathrm{C}}$ was 87.8\%. As the enrollment criteria of this experiment was based on estrus alerts from the $\mathrm{AAM}_{\mathrm{C}}$, and not by the $\mathrm{AAM}_{\mathrm{L}}$, we could not calculate a positive predictive value for the $\mathrm{AAM}_{\mathrm{L}}$. Alerts on the $\mathrm{AAM}_{\mathrm{C}}$ that were false were found to have reduced estrous expression in comparison with true positive alerts. The duration of false alert events was much shorter than true positive estrus events $(4.9 \pm 0.6$ vs. $11.8 \pm 0.3 ; P<0.001)$ and had a much smaller peak of activity $(48.4 \pm 2.1$ vs. $75.7 \pm 0.9 ; P<0.001)$. The odds of estrus events that had a short duration or a smaller peak activity to be a false alert were 12.5 and 17.6 times greater, respectively, than those with a long duration or greater peak activity $(P<0.001)$.

Additionally, stage of lactation, parity, and BCS also affected the odds of false alerts, but no effect of GS was found. Cows in early lactation had the smallest odds of showing false alerts, as both those in mid and late lactation had greater odds of showing false alerts $(P=0.04)$. Primiparous cows had greater odds than multiparous cows $(P=0.04)$ to show false alerts and a tendency for BCS to affect false alerts was found. Thin cows had the smallest odds of showing false alerts in relation to average and moderately body conditioned cows $(P=0.06)$. Odds ratios for all potential impactors of false alerts are summarized in Table 3 .

\section{Estrous Expression and Fertility}

Estrous expression when using the $\mathrm{AAM}_{\mathrm{C}}$, was found to be affected by parity, BCS, and stage of lactation. 


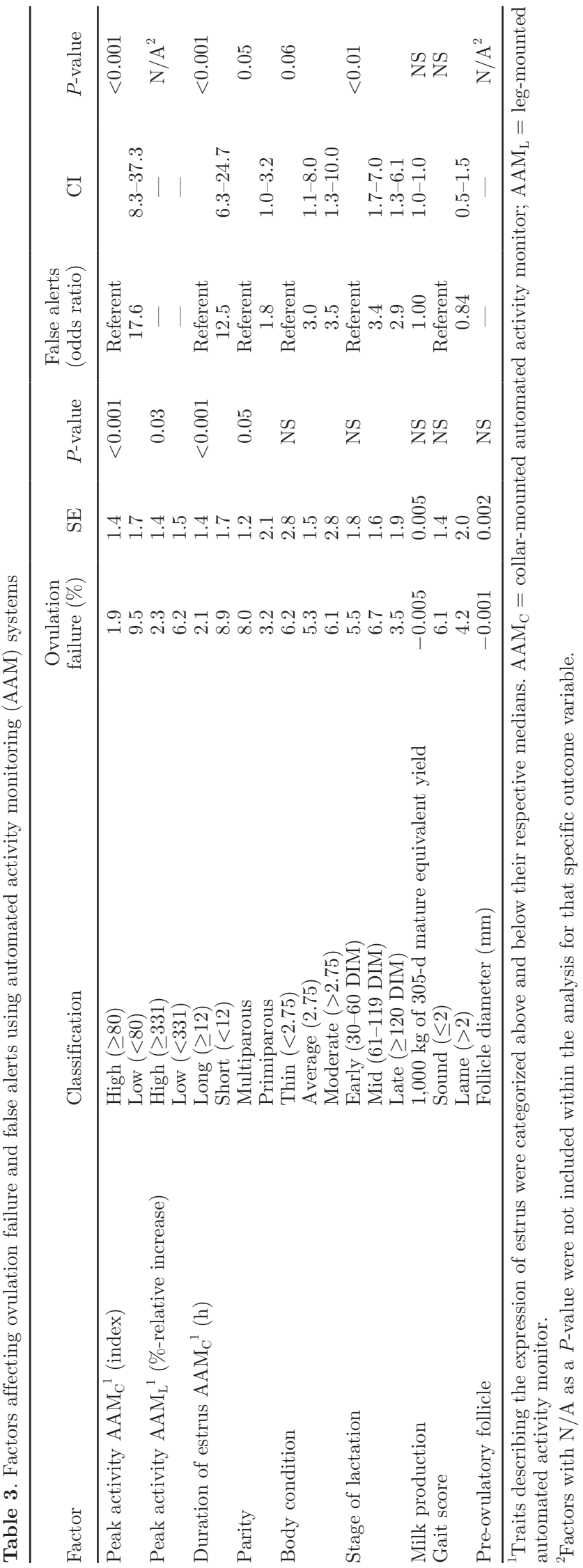

Primiparous cows had greater peak activity (77.5 \pm 1.5 vs. $73.4 \pm 0.9$ index; $P=0.02)$ and longer duration $(12.7 \pm 0.4$ vs. $11.5 \pm 0.3 \mathrm{~h} ; P=0.03)$ estrus events than multiparous cows. Thin cows had a tendency to have less activity than both average and moderately body conditioned cows $(72.3 \pm 2.0$ vs. $77.0 \pm 1.1$ vs. $77.0 \pm 1.4$ index; $P=0.08$ ); no effects of BCS were found on the duration of estrus. Events of estrus were found to have a shorter duration earlier in lactation than during mid and late lactation $(11.3 \pm 0.4$ vs. 12.6 \pm 0.4 vs. $12.4 \pm 0.4 ; P=0.02)$; no effect of stage of

A)

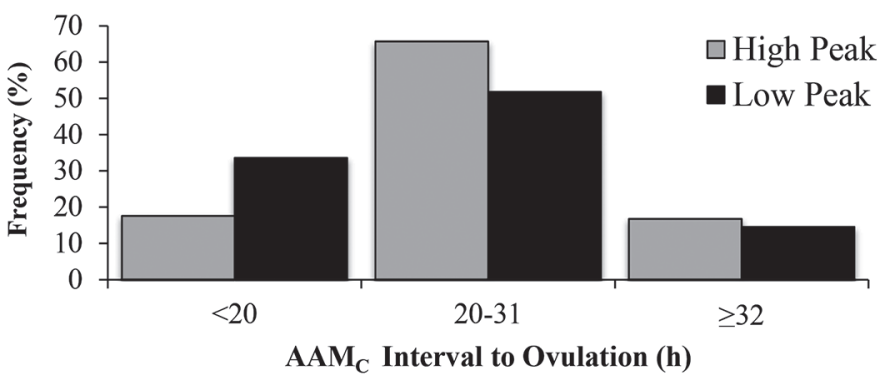

B)

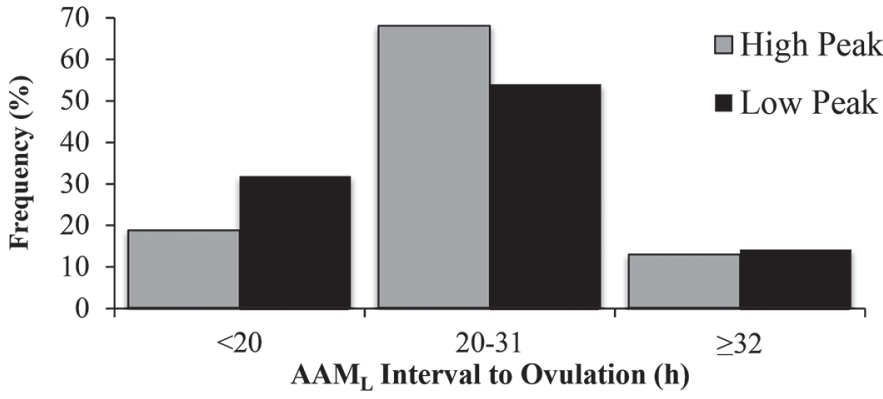

C)

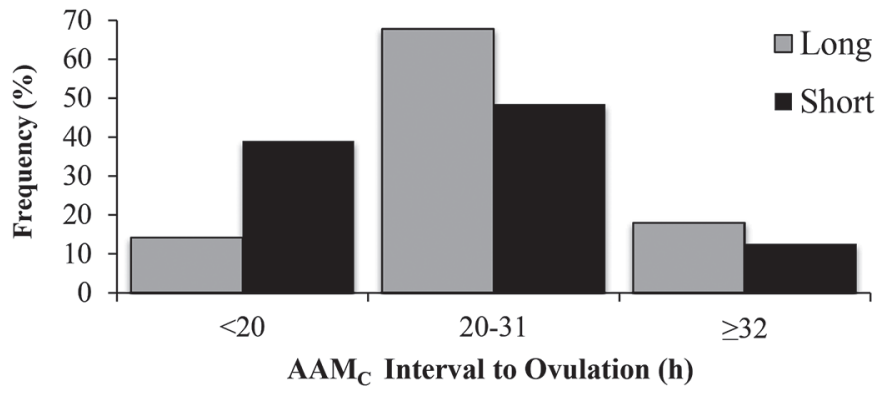

Figure 1. Frequency of estrus events for estrus alert to ovulation intervals relative to peak activity of estrus on the collar-mounted automated activity monitor $\left(\mathrm{AAM}_{\mathrm{C}}\right.$, panel $\left.\mathrm{A} ; P<0.001\right)$ and the leg-mounted automated activity monitor $\left(\mathrm{AAM}_{\mathrm{L}}\right.$; panel B; $\left.P<0.01\right)$, and duration of estrus on the $\mathrm{AAM}_{\mathrm{C}}$ (panel C; $P<0.001$ ). Peak activity was defined as the maximum activity during an episode of estrus. Duration of estrus was defined as the time the activity of the cow exceeded threshold values set by the automated activity monitor (AAM) software. Peak activity and duration of estrus were classified above and below their respective medians relative to the AAM (80 index, $331 \%$-relative increase, and $12 \mathrm{~h}$ for $\mathrm{AAM}_{\mathrm{C}}$ peak activity, $\mathrm{AAM}_{\mathrm{L}}$ peak activity, and $\mathrm{AAM}_{\mathrm{C}}$ duration, respectively). 
lactation was found on peak activity. No effects of GS, milk production, or the size of the pre-ovulatory follicle were found on the expression of estrus using the $\mathrm{AAM}_{\mathrm{C}}$; estrous expression using the $\mathrm{AAM}_{\mathrm{L}}$ was not affected by any of the variables used.

Expression of estrus also influenced fertility associated with each episode of estrus. Estrus events with greater peak activity on the $\mathrm{AAM}_{\mathrm{C}}$ and $\mathrm{AAM}_{\mathrm{L}}$ were found to have greater pregnancy per AI $(\mathbf{P} / \mathbf{A I})$ than those with less activity $\left(\mathrm{AAM}_{\mathrm{C}}: 42.3 \pm 0.4\right.$ vs. $31.7 \pm$ $0.4 \% ; P=0.02 ; \mathrm{AAM}_{\mathrm{L}}: 43.1 \pm 0.4$ vs. $36.3 \pm 0.4 \% ; P$ $=0.10)$. Furthermore, estrus events with long duration on the $\mathrm{AAM}_{\mathrm{C}}$ were also found to have greater $\mathrm{P} / \mathrm{AI}$ than those with short duration $(42.4 \pm 0.4$ vs. $32.6 \pm$ $0.4 \% ; P=0.03)$. When failed ovulations were excluded from the analysis, as events with less estrous expression are shown to have more ovulation failure than those with greater, estrous expression was still found to have a large effect on $\mathrm{P} / \mathrm{AI}$ when using the $\mathrm{AAM}_{\mathrm{C}}$ but not for the $\mathrm{AAM}_{\mathrm{L}}$ (Table 4). Parity and GS were also found to affect $\mathrm{P} / \mathrm{AI}$, whereas stage of lactation, BCS, milk production, and the size of the pre-ovulatory follicle were not. Factors affecting pregnancy per AI have been summarized in Table 4.

\section{DISCUSSION}

Factors that influence differences in timing of ovulation relative to the onset of estrus may be useful for making more informed on-farm breeding decisions. This study demonstrates that intervals from activity alerts, using AAM, to ovulation were affected by estrous expression, stage of lactation, parity, and lameness. Furthermore, we were also able to demonstrate that ovulation failure is influenced by estrous expression, and reiterate the importance of estrous expression on fertility, particularly as this information can become readily available with the use of AAM.

The intervals to ovulation of both AAM found in this study are similar to Valenza et al. (2012; 28.7 h), using a similar neck-mounted AAM, as well as other studies using pedometers (29.3 h; Roelofs et al., 2005a; Roelofs et al., 2006), pressure-sensing systems (27.6 h; Walker et al., 1996), and visual detection (30.6 h; Roelofs et al., 2005b). Stevenson et al. (2014) also reported similar intervals to ovulation using a pressure-sensing system $(26.4 \mathrm{~h})$ and a collar-mounted AAM $(25.7 \mathrm{~h})$. Suthar et al. (2011), on the other hand, reported a shorter interval to ovulation (20.6 h) using visual observation on tiestall-housed cows.

Generally, we found that ovulation was hastened in episodes of estrus with less estrous expression on AAM and those that occurred early in lactation but was delayed in cows that were considered lame. Previous

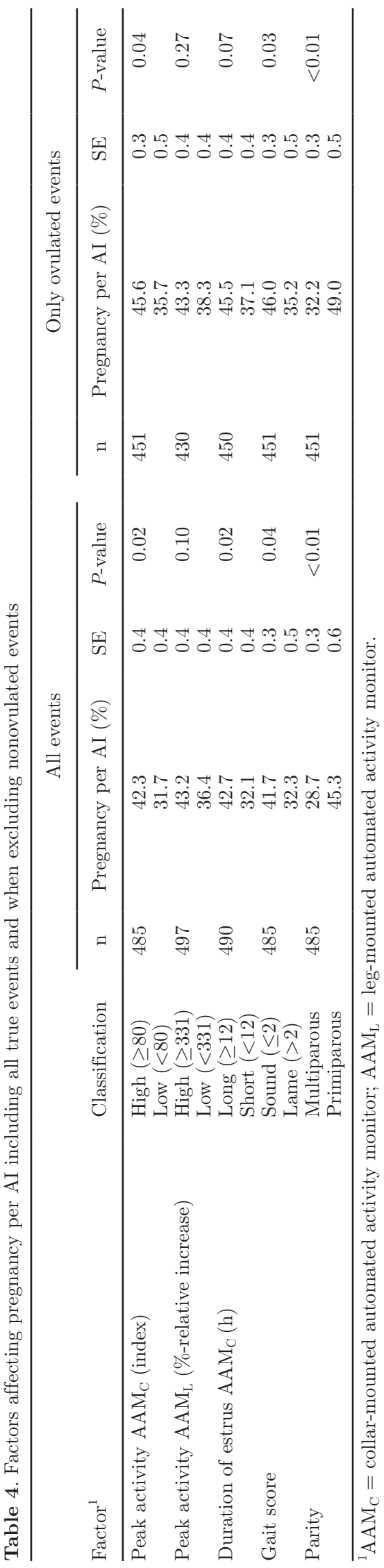


research using AAM (Hockey et al., 2010; Valenza et al., 2012; Stevenson et al., 2014), visual estrus detection (Walker et al., 1996), and pressure-sensing devices (Stevenson et al., 2014) also have reported that the interval from the onset of estrus to ovulation increased with longer duration episodes of estrus; however, this relationship has not often been related to the intensity of estrous expression. It has been suggested that differences in intervals from onset of activity to ovulation may be due to the timing of the LH surge relative to the onset of estrus behaviors (Bloch et al., 2006). Bloch et al. (2006) reported that cows with extended estrus to ovulation intervals also had lesser concentrations of estradiol at the beginning of estrus, even though there was no difference in pre-ovulatory follicle diameter, and suggest that these lesser concentrations of estradiol may be why the LH surge is delayed. Walker et al. (1996) suggested that estrus events with longer duration and thus longer intervals to ovulation may occur because circulating estradiol concentrations are adequate to cross the threshold needed for estrous expression but insufficient for ovulation. In addition, Kaim et al. (2003) noted that within a subset of cows that had severely delayed ovulation $(>50 \mathrm{~h}$ after onset of estrus), cows had significantly smaller LH surges in comparison with cows that ovulated within a more normal range. Although late and small magnitude LH surges have been associated with drastically delayed ovulation (Kaim et al., 2003) and in the case of lame cows (Dobson et al., 2007), the effect of estrous expression on the LH surge has not been fully elucidated. The occurrence of ovulation during a more ideal timing relative to insemination may be why episodes of estrus with greater estrous expression have been reported as being more fertile, both in this and previous studies (Madureira et al., 2015; Burnett et al., 2017; Silper et al., 2017).

Moreover, a recent study by Madureira et al. (2015) found a poor association between estradiol concentration measured at the time of alert and the intensity of estrous expression. Saumande and Humblot (2005) and Hockey et al. (2010) noted a correlation with the size of the ovulatory follicle and the interval from onset of activity to ovulation; however, both Van Eerdenburg et al. (2002) and Madureira et al. (2015) reported no influence of pre-ovulatory follicle size on estrous expression. Similarly, Bloch et al. (2006) reported no differences in pre-ovulatory follicle size among estrus to ovulation interval lengths. In the present study, estradiol was not measured, but no effect of the pre-ovulatory follicle diameter on the interval from onset of estrus to ovulation or on estrous expression was found. Further research is needed to determine which physiological events can account for the changes in the interval from activity alert to ovulation associated with estrous expression.

Estrous expression also had a large effect on ovulation failure and fertility regardless of which AAM was used. Although cows with greater estrous expression had significantly less ovulation failure (3.5 vs. $11.5 \%$ ), it is probably not the only explanation for the differences seen in $\mathrm{P} / \mathrm{AI}$ between cows that express a greater or lesser intensity of estrous behaviors in this study. When removing cows that did not ovulate, the same pattern of increased $\mathrm{P} / \mathrm{AI}$ at events of estrus with greater estrous expression was maintained overall, suggesting that other processes (e.g., uterine environment) must be at play to explain the changes in fertility observed herein.

Similar to the current study, Valenza et al. (2012) reported $95 \%$ of cows that were both in estrus and indicated by an alert on an AAM successfully ovulated. In another study that used progesterone to discriminate between true and false estrus alerts on an AAM, they reported that $91.5 \%$ of cows that had an alert with low progesterone $(<1 \mathrm{ng} / \mathrm{mL})$ successfully ovulated, and within that $85.5 \%$ ovulated $<36 \mathrm{~h}$ after the alert (Stevenson et al., 2014). Others have reported similar ovulation failure rates $(6.5 \%)$ as well as an effect of season (12.4\% warm season; $3.4 \%$ cool season; LópezGatius et al., 2005), although the current study did not observe a similar seasonal effect. Ovulation failure is generally thought to result from a lack of responsiveness of the hypothalamus to increased circulating concentrations of estradiol and thus failure to trigger the GnRH surge and ultimately the LH surge (Wiltbank et al., 2002). Factors affecting ovulation failure are mainly those associated with infection and stress. For example, heat stress (López-Gatius et al., 2005), uterine infection (Sheldon et al., 2009), and ACTH (Ribadu et al., 1999, 2000) or endotoxin treatments (Peter et al., 1989) are all stress factors associated with ovulation failure. Although not all anovulation is caused by cystic ovarian follicles (Wiltbank et al., 2002; Vanholder et al., 2006), additional risk factors of cystic ovarian follicles have been reported as milk production (Laporte et al., 1994; Heuer et al., 1999), parity (Laporte et al., 1994), and stage of lactation (Vanholder et al., 2006). Moreover, cystic cows have been reported as having a lack of standing estrus, or frequent bouts of estrual behaviors (Wiltbank et al., 2002) but very few reports have been done on this subject. To the best of our knowledge, this is the first study to report the effects of estrous expression (i.e., intensity and duration) using an AAM on ovulation failure.

Stage of lactation was found to affect estrous expression and the interval from activity alert to ovulation, 
but not ovulation failure or fertility. Effects of stage of lactation on estrous expression could be from many different factors, including the number of estrous cycles that have occurred since parturition (Lopez et al., 2004; Aungier et al., 2012), the amount of milk being produced (Lopez et al., 2004), or the presence of negative energy balance (Butler, 2000). Effects of BCS on estrous expression may be symptomatic of negative energy balance. Previous research has demonstrated the harmful effects of negative energy balance and poor BCS on fertility (Butler, 2000; Moreira et al., 2000) and estrous expression (Madureira et al., 2015). In contrast, in the present study, we are unable to decipher which of these factors related to stage of lactation are most influential on estrous expression. Previous research also supports that stage in lactation may not have a key effect on ovulation failure (López-Gatius et al., 2005). Conversely, Demetrio et al. (2007) found cows with increased DIM had a greater chance of ovulation failure. Negative energy balance postpartum has been shown to decrease pulsatile LH secretion and IGF-1 concentrations (Diskin et al., 2003), which are important for follicular growth as well as ovulation; however, the lack of effect of stage of lactation on ovulation failure also may have resulted from the exclusion of estrus events that occurred before 30 DIM.

Parity was also found to be an important factor that affected ovulation failure, estrous expression, and fertility, but not intervals to ovulation. Other research has also reported primiparous cows to have longer duration episodes of estrus and to take more total steps per estrus using pedometers (Roelofs et al., 2005a), and express more behaviors at estrus when using visual observation (Roelofs et al., 2005a,b). In contrast, Van Eerdenburg et al. (2002) found no effects of parity on behavioral scoring, nor did Valenza et al. (2012) and Stevenson et al. (2014) find differences in estrus duration using AAM. Similar to the current study, Roelofs et al. (2005a,b) and Walker et al. (1996) found no difference in interval from the beginning of estrus to ovulation between multiparous and primiparous cows using pedometers and the visual detection of estrus, whereas Stevenson et al. (2014) found primiparous cows to have shorter ovulation intervals from onset of activity. In contrast, Roelofs et al. (2005a,b) did find that primiparous cows had a shorter interval from the end of estrus to ovulation when using pedometers (Roelofs et al., 2005a). The difference in the interval from the end of estrus to ovulation was speculated to be the result of greater duration of estrus in primiparous cows.

The current study was not designed to report how accurate the AAM were able to alert estrus events, but was able to determine which characteristics predispose cows to false alerts. False alerts affect reproductive per- formance through inappropriate use of labor and costs associated with inseminations of cows that are not in estrus, as well as the potential of harming a pre-existing pregnancy that may be present but not yet diagnosed. In addition, false alerts can be damaging as they can cause producers to lose trust in the technology or become frustrated, leading to decreased use of the system. Estrous expression was found to be important for deciphering false alerts, as estrus events with less estrous expression were more likely to be false than events having greater estrous expression. The current study found less estrous expression, greater BCS, later days in milk, and being primiparous to be risk factors that increased the odds of showing more false alerts than their counterparts. A previous study demonstrated that false estrus alerts had fewer actual steps when using a pedometer in comparison with correctly alerted estrus events (477 \pm 322 steps vs. $841 \pm 259$ steps); however, they did not find the same relationship when looking at mean and standard deviation of steps at estrus alert (Roelofs et al., 2005a). Similarly, a previous study reported that increased time postpartum increased the odds of a cow to demonstrate a false-positive alert (Holman et al., 2011); that study found no effect of BCS, parity GS, or milk yield on false alerts using different automated technologies as well as visual detection. Results from this study may suggest that cows that are in a healthier state (i.e., greater BCS, later in lactation) may engage in certain activities that may be more likely to cause them to be falsely identified as in estrus.

Interestingly, the factors reported by Aungier et al. (2012) to affect the ability of the AAM to correctly identify estrus are similar to those that we found to affect false alerts. Aungier et al. (2012) reported estrous expression to have a significant effect on alerts, where the odds of a correct alert increased by 20 and 91\% with every 1-unit increase in peak activity and 2-h increase duration, respectively. In addition, Aungier et al. (2012) reported effects of BCS, milk yield, and the quantity of postpartum pre-ovulatory follicular phases on the likelihood of positive alerts, but contrary to the current study did not find effects of parity on alerts. They reported that estrus events occurring at second or subsequent pre-ovulatory cycles, rather than the first postpartum, had increased odds of 8.2 for being correctly identified by the monitor and that the odds of a pre-ovulatory phase being identified by the AAM improved by 1.4 for every increase in 0.25 BCS unit. Aungier et al. (2012) also found that cows producing less milk at the time of estrus were more likely to be identified. In the current study we did not find an effect of milk yield on the occurrence of false alerts. Neither this study or Aungier et al. (2012) reported any effects of lameness on the occurrence of false or positive alerts. 


\section{CONCLUSIONS}

This study demonstrated that the expression of estrus, measured by 2 different AAM systems, is important, not only for estrus detection, but also for its effects on the interval from activity alert to ovulation, ovulation failure, and fertility. Timing of ovulation is a central factor that affects on-farm breeding decisions; thus, understanding the factors associated with it can yield important information to refine a more efficient use of AAM. In addition to estrous expression, stage of lactation and lameness were also found to influence the timing of ovulation relative to an estrus alert. Furthermore, we demonstrated that estrous expression influenced ovulation failure, although the occurrence of ovulation failure cannot solely explain the difference in fertility between estrus events with greater and lesser estrous expression.

\section{ACKNOWLEDGMENTS}

This study was supported by a contribution from the Dairy Research Cluster Initiative (Dairy Farmers of Canada, Agriculture and Agri-Food Canada, the Canadian Dairy Network, and the Canadian Dairy Commission, Ontario, Canada). Partial funding was also provided by the Natural Sciences and Engineering Research Council grant NSERC-RGPIN 418672-13 and the BC Dairy Association (Burnaby, BC, Canada). The authors also thank Nelson Dinn and all students that assisted with data collection at the University of British Columbia Dairy Education and Research Centre.

\section{REFERENCES}

Aungier, S. P. M., J. F. Roche, M. Sheehy, and M. A. Crowe. 2012. Effects of management and health on the use of activity monitoring for estrus detection in dairy cows. J. Dairy Sci. 95:2452-2466.

Bijker, I., R. M. Christley, R. F. Smith, and H. Dobson. 2015. Effect of signs of oestrus, disease stressors and cow activity on pregnancy rate following artificial insemination. Vet. Rec. 176:411-416.

Bloch, A., Y. Folman, M. Kaim, Z. Roth, R. Braw-Tal, and D. Wolfenson. 2006. Endocrine alterations associated with extended time interval between estrus and ovulation in high-yield dairy cows. J. Dairy Sci. 89:4694-4702.

Buckley, F., K. O'Sullivan, J. F. Mee, R. D. Evans, and P. Dillon. 2003. Relationships among milk yield, body condition, cow weight, and reproduction in spring-calved Holstein-Friesians. J. Dairy Sci. 86:2308-2319.

Burnett, T. A., A. M. L. Madureira, B. F. Silper, A. C. C. Fernandes, and R. L. A. Cerri. 2017. Integrating an automated activity monitor into an artificial insemination program and the associated risk factors affecting reproductive performance of dairy cows. J. Dairy Sci. 100:5005-5018.

Butler, W. R. 2000. Nutritional interactions with reproductive performance in dairy cattle. Anim. Reprod. Sci. 60-61:449-457.

Canadian Council for Animal Care. 2009. The Care and Use of Farm Animals in Research, Teaching and Testing. Canadian Council for Animal Care, Ottawa, Ontario, Canada.
Dalton, J. C., S. Nadir, J. H. Bame, M. Noftsinger, R. L. Nebel, and R. G. Saacke. 2001. Effect of time of insemination on number of accessory sperm, fertilization rate, embryo quality in nonlactating dairy cattle. J. Dairy Sci. 84:2413-2418.

Demetrio, D. G. B., R. M. Santos, C. G. B. Demetrio, and J. L. M. Vasconcelos. 2007. Factors affecting conception rates following artificial insemination or embryo transfer in lactation Holstein cows. J. Dairy Sci. 90:5073-5082.

Diskin, M. G., D. R. Mackey, J. F. Roche, and J. M. Sreenan. 2003. Effects of nutrition and metabolic status on circulating hormones and ovarian follicle development in cattle. Anim. Reprod. Sci. $78: 345-370$.

Dobson, H., R. Smith, M. Royal, C. Knight, and I. Sheldon. 2007. The high-producing dairy cow and its reproductive performance. Reprod. Domest. Anim. 42:17-23.

Dransfield, M. B. G., R. L. Nebel, R. E. Pearson, and L. D. Warnick. 1998. Timing of insemination for dairy cows identified in estrus by a radiotelemetric estrus detection system. J. Dairy Sci. 81:1874-1882.

Edmonson, A. J., I. J. Lean, L. D. Weaver, T. Farver, and G. Webster. 1989. A body condition scoring chart for Holstein dairy cows. J. Dairy Sci. 72:68-78.

Flower, F. C., and D. M. Weary. 2006. Effect of hoof pathologies on subjective assessments of dairy cow gait. J. Dairy Sci. 89:139-146.

Hall, J. G., C. Branton, and E. J. Stone. 1959. Estrus, estrous cycles, ovulation time, time of service and fertility of dairy cattle in Louisiana. J. Dairy Sci. 42:1086-1094.

Hernandez, J., J. K. Shearer, and D. W. Webb. 2001. Effect of lameness on the calving-to-conception interval in dairy cows. J. Am. Vet. Med. Assoc. 218:1611-1614.

Heuer, C., Y. H. Schukken, and P. Dobbelaar. 1999. Post-partum body condition score and results from the first test day milk yield as predictors of disease, fertility, yield, and culling in commercial dairy herds. J. Dairy Sci. 82:295-304.

Hockey, C., J. Morton, S. Norman, and M. McGowan. 2010. Evaluation of a neck mounted 2-hourly activity meter system for detecting cows about to ovulate in two paddock-based Australian dairy herds. Reprod. Domest. Anim. 45:e107-e117.

Holman, A., J. Thompson, J. E. Routly, J. Cameron, D. N. Jones, R. F. Smith, and H. Dobson. 2011. Comparison of oestrus detection methods in dairy cattle. Vet. Rec. 169:47-52.

Kaim, M., A. Bloch, D. Wolfenson, R. Braw-Tal, M. Rosenberg, H. Voet, and Y. Folman. 2003. Effects of GnRH administered to cows at the onset of estrus on timing of ovulation, endocrine responses, and conception. J. Dairy Sci. 86:2012-2021.

Laporte, H. M., H. Hogeveen, Y. H. Schukken, and J. P. T. M. Noordhuizen. 1994. Cystic ovarian disease in Dutch dairy cattle I. Incidence, risk factors and consequences. Livest. Prod. Sci. 38:191-197.

Lopez, H., L. D. Satter, and M. C. Wiltbank. 2004. Relationship between level of milk production and estrous behavior of lactating dairy cows. Anim. Reprod. Sci. 81:209-223.

López-Gatius, F., M. López-Béjar, M. Fenech, and R. H. F. Hunter. 2005. Ovulation failure and double ovulation in dairy cattle: Risk factors and effects. Theriogenology 63:1298-1307.

Madureira, A. M. L., B. F. Silper, T. A. Burnett, L. Polsky, L. H Cruppe, D. M. Veira, J. L. M. Vasconcelos, and R. L. A. Cerri. 2015. Factors affecting expression of estrus measured by activity monitors and conception risk of lactating dairy cows. J. Dairy Sci. 98:7003-7014.

Melendez, P., J. Bartolome, L. F. Archbald, and A. Donovan. 2003. The association between lameness, ovarian cysts and fertility in lactating dairy cows. Theriogenology 59:927-937.

Moreira, F., C. Risco, M. F. A. Pires, J. D. Ambrose, M. Drost, M. DeLorenzo, and W. W. Thatcher. 2000. Effect of body condition on reproductive efficiency of lactating dairy cows receiving a timed insemination. Theriogenology 53:1305-1319.

NRC. 2001. Nutrient Requirements of Dairy Cattle. 7th rev. ed. Natl. Acad. Sci., Washington, DC.

Peter, A. T., W. T. K. Bosu, and R. J. DeDecker. 1989. Suppression of preovulatory luteinizing hormone surges in heifers after intra- 
uterine infusions of Escherichia coli endotoxin. Am. J. Vet. Res. $50: 368-373$.

Ribadu, A. Y., K. Nakada, M. Moriyoshi, W. C. Zhang, Y. Tanaka, and T. Nakao. 2000. The role of LH pulse frequency in ACTHinduced ovarian follicular cysts in heifers. Anim. Reprod. Sci. 64:21-31.

Ribadu, A. Y., K. Nakada, Y. Tanaka, M. Moriyoshi, W. C. Zhang, and T. Nakao. 1999. Lack of LH response to exogenous estradiol in heifers with ACTH-induced ovarian follicular cysts. J. Vet. Med. Sci. 61:979-981.

Rivera, F., C. Narciso, R. Oliveira, R. L. A. Cerri, A. Correa-Calderón, R. C. Chebel, and J. E. P. Santos. 2010. Effect of bovine somatotropin $(500 \mathrm{mg}$ ) administered at ten-day intervals on ovulatory responses, expression of estrus, and fertility in dairy cows. J. Dairy Sci. 93:1500-1510.

Roche, J. R., K. A. Macdonald, C. R. Burke, J. M. Lee, and D. P. Berry. 2007. Associations among body condition score, body weight, and reproductive performance in seasonal-calving dairy cattle. J. Dairy Sci. 90:376-391.

Roche, J. R., N. C. Friggens, J. K. Kay, M. W. Fisher, K. J. Stafford and D. P. Berry. 2009. Invited review: Body condition score and its association with dairy cow productivity, health, and welfare. J. Dairy Sci. 92:5769-5801.

Roelofs, J. B., E. A. M. Graat, E. Mullaart, N. M. Soede, W. Voskamp-Harkema, and B. Kemp. 2006. Effects of insemination-ovulation interval on fertilization rates and embryo characteristics in dairy cattle. Theriogenology 66:2173-2181.

Roelofs, J. B., F. J. van Eerdenburg, N. M. Soede, and B. Kemp. 2005a. Pedometer readings for estrous detection and as predictor for time of ovulation in dairy cattle. Theriogenology 64:1690-1703.

Roelofs, J. B., F. J. van Eerdenburg, N. M. Soede, and B. Kemp. 2005b. Various behavioral signs of estrous and their relationship with time of ovulation in dairy cattle. Theriogenology 63:13661377.

Saacke, R. G., J. C. Dalton, S. Nadir, R. L. Nebel, and J. H. Bame. 2000. Relationship of seminal traits and insemination time to fertilization rate and embryo quality. Anim. Reprod. Sci. 60-61:663677.
Saumande, J., and P. Humblot. 2005. The variability in the interval between estrus and ovulation in cattle and its determinants. Anim. Reprod. Sci. 85:171-182.

Sheldon, I. M., J. Cronin, L. Goetze, G. Donofrio, and H. J. Schuberth. 2009. Defining postpartum uterine disease and the mechanisms of infection and immunity in the female reproductive tract in cattle. Biol. Reprod. 81:1025-1032.

Silper, B. F., A. M. L. Madureira, L. B. Polsky, S. Soriano, A. F. Sica, J. L. M. Vasconcelos, and R. L. A. Cerri. 2017. Daily lying behavior of lactating Holstein cows during an estrus synchronization protocol and its associations with fertility. J. Dairy Sci. 100:8484-8495

Stevenson, J. S. 2001. Reproductive management of dairy cows in high milk-producing herds. J. Dairy Sci. 84(E. Suppl.):E128-E143.

Stevenson, J. S., S. L. Hill, R. L. Nebel, and J. M. DeJarnette. 2014 Ovulation timing and conception risk after automated activity monitoring in lactating dairy cows. J. Dairy Sci. 97:4296-4308.

Suthar, V. S., O. Burfeind, J. S. Patel, A. J. Dhami, and W. Heuwieser. 2011. Body temperature around induced estrus in dairy cows. J. Dairy Sci. 94:2368-2373.

Valenza, A., J. O. Giordano, G. Lopes Jr., L. Vincenti, M. C. Amundson, and P. M. Fricke. 2012. Assessment of an accelerometer system for detection of estrus and treatment with gonadotropin-releasing hormone at the time of insemination in lactating dairy cows. J. Dairy Sci. 95:7115-7127.

Van Eerdenburg, F. J. C. M., D. Karthaus, M. A. M. Taverne, I. Mercis, and O. Szenci. 2002. The relationship between estrous behavioral score and time of ovulation in dairy cattle. J. Dairy Sci. 85:1150-1156.

Vanholder, T., G. Opsomer, and A. De Kruif. 2006. Aetiology and pathogenesis of cystic ovarian follicles in dairy cattle: A review. Reprod. Nutr. Dev. 46:105-119.

Walker, W. L., R. L. Nebel, and M. L. McGilliard. 1996. Time of ovulation relative to mounting activity in dairy cattle. J. Dairy Sci. 79:1555-1561.

Wiltbank, M. C., A. Gümen, and R. Sartori. 2002. Physiological classification of anovulatory conditions in cattle. Theriogenology $57: 21-52$. 